\title{
A CONSTRUCTION OF LIE ALGEBRAS BY TRIPLE SYSTEMS
}

\author{
BY
}

W. HEIN

\begin{abstract}
A construction of Lie algebras by means of special unital representations of Jordan algebras on a certain kind of triple systems is given which generalizes the construction due to Freudenthal, Faulkner and Koecher.
\end{abstract}

Introduction. A common feature of all the Lie algebras obtained by either the Freudenthal, Faulkner or Koecher construction (cf. [4] , [3] , [7] resp.) is that there is an $X$ in any such algebra $\mathbb{R}$ such that the eigenvalues of ad $X$ are contained in the set $\{0, \pm 1, \pm 1 / 2\}$ (cf. [3], [4], [7]). Moreover, the direct sum of the +1 and $-1 / 2$ eigenspace turns out to be a triple system (a vector space together with a trilinear inner composition) such that $\mathfrak{R}$ decomposes as the vector space direct sum $\Re(\mathfrak{T}) \oplus \mathfrak{I} \oplus \overline{\mathfrak{T}}$ where $\Re(\mathfrak{T})$ is a subalgebra of the Lie algebra of all linear transformations on $\mathfrak{T}$ and $\overline{\mathfrak{F}}$ is another copy of $\mathfrak{I}$. If $\mathfrak{R}$ is obtained by the Koecher construction, the $\pm 1 / 2$ eigenspaces are $\{0\}$; if $\mathfrak{R}$ is obtained by the method of Faulkner, the \pm 1 eigenspaces are one dimensional. Consulting a table of roots of the simple Lie algebras over an algebraically closed field of characteristic zero one finds that each such Lie algebra contains an element $X$ such that the eigenspaces of ad $X$ do not satisfy the above dimension restrictions. This is the point where the present paper starts.

In $\S 1$, we define an anticommutative algebra structure on the vector space $\mathfrak{R}(\mathfrak{T}) \oplus \mathfrak{T} \oplus \overline{\mathfrak{T}}$ where $\mathfrak{T}$ is a certain triple system, $\overline{\mathfrak{T}}$ another copy of $\mathfrak{T}$ and $\mathfrak{N}(\mathfrak{T})$ a Lie algebra of linear transformations of $\mathfrak{T}$. Assuming the Jacobi identity to be satisfied we deduce a set of identities which describes $\mathfrak{T}$ by a Jordan sub triple system $\mathfrak{U}$ (see [7] or [8] for definition) and a certain triple system $B$, a special representation $\sigma$ of the Jordan triple system $\mathcal{U}$ on the underlying vector space of $B$ and a bilinear mapping from $B \times B$ to $\mathscr{A}$ ( $f$ is not needed if $\sigma$ is faithful). We restrict ourselves to the case where $\mathcal{U}$ is

Received by the editors September 7, 1973.

AMS (MOS) subject classifications (1970). Primary 17B05, 17B25, 17C15; Secondary $08 \mathrm{A05}$.

Key words and phrases. Lie algebras, Jordan algebras, special representations of Jordan algebras, triple systems. 
a Jordan algebra with unit element. Then $\mathscr{Q}$ is the +1 and $B$ is the $-1 / 2$ eigenspace of ad $X$ with suitable $X$ in $\Re(\mathfrak{T})$.

In $\S 2$, we start with a situation $\mathcal{Q}, \sigma, f, B$ which satisfies certain axioms and make the vector space direct sum $\mathfrak{T}=\mathscr{U} \oplus \mathbb{B}$ a triple system such that the construction of $\S 1$, yields a Lie algebra $\mathfrak{R}(\mathfrak{T})$.

We investigate the ideals of $\mathfrak{T}$ and $\mathfrak{R}(\mathfrak{T})$ in $\S 3$ and give conditions for $\mathbb{T}$ and $\mathfrak{R}(\mathfrak{T})$ to be simple.

In $\S 4$, we give a set of examples which provide Lie algebras of type $B, C$, and $D$.

Using symplectic representations of Jordan algebras and Lie algebras we construct in $\$ 5$ triple systems as considered in $\$ 2$, such that Lie algebras of type $G_{2}, F_{4}$ and $E_{8}$ are obtained, the latter two types by means of spin representations of Clifford algebras.

Note. After the present paper had been completed, the author was referred by J. R. Faulkner to a paper of I. L. Kantor in Soviet Math. Dokl. 14 (1973), pp. 254-258, who gives a construction which is related to that given in the present paper.

According to the referee, another related paper is A construction of Lie algebras from $\Im$-ternary algebras, by B. N. Allison, which will appear in Amer. J. Math.

1. A class of anticommutative algebras.

1.1. Let $A, B$ be vector spaces over a field of characteristic not two, $A \neq\{0\}$. Suppose that on the direct sum $T=A \oplus B$ a trilinear inner composition $(u, v, w) \mapsto\langle u v w\rangle$ is defined satisfying

$$
\langle T T A\rangle \subset A, \quad\langle T T B\rangle \subset B,
$$

$$
\langle A B T\rangle=\langle B A T\rangle=\{0\},
$$

and that there is an element $e \neq 0$ in $A$ such that

$$
\langle a b e\rangle+\langle b a e\rangle=0
$$

for all $a, b \in B$.

Let $\mathfrak{I}$ denote the triple system $(T,\langle\rangle)$ and $\Re(\mathfrak{T})$ the Lie algebra of linear transformations on $\mathfrak{T}$ which is generated by the transformations $L(u, v)$, where $L(u, v)$ is defined for $u, v \in \mathfrak{T}$ by $L(u, v) w:=\langle u v w\rangle$, $w \in \mathfrak{I}$.

Let $\bar{A}, \bar{B}$ be other copies of $A$ and $B$ resp. and let $x \mapsto \bar{x}, a \mapsto \bar{a}$ be isomorphisms of $A$ onto $\bar{A}$ and $B$ onto $\bar{B}$ resp.

In order to construct an anticommutative algebra, we define, using an 
involution $N \mapsto N^{*}$ of the Lie algebra $\mathfrak{N}(\mathfrak{T})$, a composition on the vector space direct sum $\mathfrak{R}(\mathfrak{I}) \oplus A \oplus \bar{A} \oplus B \oplus \bar{B}$ by

$$
\begin{aligned}
{\left[N, N^{\prime}+x+\bar{y}+a+\bar{b}\right] } & :=\left[N, N^{\prime}\right]+N x-\overline{N^{*} y}+N a-\overline{N^{*} b}, \\
{[x, y+\bar{z}+a+\bar{b}] } & :=2 L(x, z)-2 \overline{\langle x e a\rangle} \\
{[\bar{x}, \bar{y}+a+\bar{b}] } & :=-2\langle x e b\rangle \\
{[a, b+\bar{c}] } & :=L(a, c)+\overline{\langle a b e\rangle} \\
{[\bar{a}, \bar{b}] } & :=-\langle a b e\rangle,
\end{aligned}
$$

for $N, N^{\prime} \in \Re(\mathfrak{T}), x, y, z \in A$ and $a, b, c \in B$. The remaining products are then defined by anticommutativity, which makes sense because of (2). We denote this algebra by $\mathfrak{R}\left(\mathfrak{T}^{*}{ }^{*}\right)$. Put $L_{0}:=\Re(\mathfrak{T}), L_{1}:=A, L_{-1}:=\bar{A}, L_{-1 / 2}:=B$ and $L_{1 / 2}:=\bar{B}$. Then (1) and (2) yield $\left[L_{\nu}, L_{\mu}\right] \subset L_{\nu+\mu}$ for $\nu, \mu=0, \pm 1, \pm 1 / 2$ (it being understood that $L_{\nu+\mu}=\{0\}$ if $\nu+\mu$ is different from $0, \pm 1$ and $\pm 1 / 2)$.

1.2. Obviously, the anticommutative algebra just defined is a Lie algebra if and only if the Jacobi identity

$$
J(X, Y, Z):=[X,[Y, Z]]+[Y,[Z, X]]+[Z,[X, Y]]=0
$$

is satisfied for all $X, Y, Z \in \mathfrak{R}\left(\mathfrak{F},{ }^{*}\right)$. Since $\mathfrak{\Re ( T )}$ is a Lie algebra, $J\left(N_{1}, N_{2}, N_{3}\right)$ $=0$ holds for $N_{i} \in \Re(\mathfrak{T})$. Since $N \mapsto N^{*}$ is an involution, $J\left(N_{1}, N_{2}, X\right)$ $=0$ holds for $N_{i} \in \mathfrak{R}(\mathfrak{T})$ and $X \in \mathfrak{R}\left(\mathfrak{X},{ }^{*}\right)$. Using the fact that the mapping $J$ is alternating, a simple computation shows that $\mathfrak{L}\left(\mathfrak{T},{ }^{*}\right)$ is a Lie algebra if and only if the following identities are satisfied for all $N \in \mathfrak{N}(\mathfrak{T}), u, v \in \mathfrak{T}$, $x, y, z \in A$ and $a, b, c \in B$ :
(a) $[N, L(u, v)]=L(N u, v)-L\left(u, N^{*} v\right)$,
(b) $\langle x y z\rangle=\langle z y x\rangle$,
(c) $\langle a b c\rangle=\langle c b a\rangle-2\langle\langle a c e\rangle e b\rangle$,
(d) $\langle x y a\rangle=-2\langle y e\langle x e a\rangle\rangle$,
(e) $\langle a b x\rangle=-2\langle\langle x e a\rangle b e\rangle$,
(f) $N^{*}\langle x e a\rangle+\langle(N x) e a\rangle+\langle x e(N a)\rangle=0$,
(g) $N^{*}\langle a b e\rangle+\langle(N a) b e\rangle+\langle a(N b) e\rangle=0$,
(h) $L(x,\langle a b e\rangle)+L(a,\langle x e b\rangle)-L(b,\langle x e a\rangle)=0$,
(i) $L(x, y)^{*} z=L(x, z)^{*} y$,
(k) $L(a, b)^{*} c=L(a, c)^{*} b+2\langle\langle b c e\rangle e a\rangle$,
(l) $L(x, y)^{*} a=-2\langle x e\langle y e a\rangle$,
(m) $L(a, b)^{*} x=2\langle\langle x e b\rangle a e\rangle$,
(n) $L(\langle a b e\rangle, x)+L(\langle x e a\rangle, b)-L(\langle x e b\rangle, a)=0$.

1.3. In view of the next section we remark 
a. If $L(x, y)^{*}=L(y, x)$ and $L(a, b)^{*}=-L(b, a)$ hold for $x, y \in A$ and $a, b \in B$, the identities (i)-(n) follow from the former ones.

b. Suppose $L(x, y)^{*}=L(y, x)$ holds for all $x, y \in A$ and (a)-(h) are satisfied. Then (a) and (b) imply that $A$ together with the composition $\langle x y z\rangle$ is a Jordan triple system (cf. [8]). From (d) and (f) one concludes immediately that

$$
\langle\langle x y z\rangle e a\rangle=2(\langle x e\langle y e\langle z e a\rangle\rangle\rangle+\langle z e\langle y e\langle x e a\rangle\rangle\rangle)
$$

for $x, y, z \in A$ and $a \in B$. With $\sigma(x):=-2 L(x, e)_{\mid B}, x \in A$, this can be rewritten as

$$
\sigma(\langle x y z\rangle)=1 / 2(\sigma(x) \sigma(y) \sigma(z)+\sigma(z) \sigma(y) \sigma(x))
$$

which means that $\sigma$ is a special representation of the Jordan triple system $(A,\langle\rangle)$. If the characteristic of the groundfield is not three, then $A$ is a Jordan algebra under the composition $x \cdot y=\langle x e y\rangle$ (cf. $[8$, p. 70]) and $\sigma$ is a special representation of this Jordan algebra. If in addition $\langle e e x\rangle=x$ for all $x \in A$ (that means $e$ is the unit element of $(A, \cdot)$ ), then (a) yields

$$
\langle x y z\rangle=(x y) z+x(y z)-y(x z)
$$

for $x, y, z \in A$.

c. Suppose $B=\{0\}$. Then (1) is satisfied and (2) holds for all $e \in A$. The composition rule can now be written as

$$
\begin{aligned}
{\left[N, N^{\prime}+x+\bar{y}\right] } & =\left[N, N^{\prime}\right]+N x-\overline{N^{*} y}, \\
{[x, y+\bar{z}] } & =2 L(x, z), \\
{[\bar{x}, \bar{y}] } & =0
\end{aligned}
$$

for $N, N^{\prime} \in \mathfrak{N}(\mathfrak{Y})$ and $x, y, z \in A$. Thus, in the case where $L(x, y)^{*}=$ $L(y, x)$ for $x, y \in A$ our construction is the same as that given by Meyberg in [8]. If in addition $A$ is a Jordan algebra under the composition $x \cdot y=$ 〈xey > with unit element $e$ we observe, using (4), that our construction is identical with the Koecher construction (cf. [6]).

2. A class of triple systems. In this section we shall be interested in those triple systems used in $\S 1$ to construct the anticommutative algebra $\mathfrak{R}\left(\mathfrak{T},{ }^{*}\right)$. We restrict ourselves to the case where $A$ with the multiplication $x \cdot y=$ $\langle x e y\rangle$ is a Jordan algebra with unit element $e$ (see 1.3b). We describe the triple system $\mathfrak{T}$ in terms of a Jordan algebra $\mathcal{U}$, a certain kind of triple system $\$$, a special unital representation $\sigma$ of $\mathcal{U}$ on the underlying vector space of $B$ 
and a skew symmetric bilinear mapping from $B \times B$ to $\mathscr{Q}$ by a system of axioms, which guarantee that with a suitable involution * of the Lie algebra $\Re(\mathfrak{F})$ of endomorphisms of $\mathfrak{T}$ generated by the linear mappings $w \mapsto\langle u v w\rangle$ the algebra $\mathfrak{R}\left(\mathfrak{T},{ }^{*}\right)$ is a Lie algebra.

2.1. Let it be a Jordan algebra with unit element $e \neq 0$ over a field $F$ of characteristic not two and $\sigma$ a special unital representation of $\mathcal{U}$ on the vector space $B$ over $F$, that means a $F$-linear mapping from $A$ to $\operatorname{End}_{F}(B)$ satisfying

$$
\sigma(x y)=1 / 2(\sigma(x) \sigma(y)+\sigma(y) \sigma(x))
$$

and

$$
\sigma(e)=\mathrm{id}_{B}
$$

for all $x, y \in \mathfrak{Z}$. We denote $\sigma(x) a$ by $x$. a.

Let $f$ be a skew symmetric bilinear mapping from $B \times B$ to $\mathscr{U}$ such that

$$
2 x f(a, b)=f(x \cdot a, b)+f(a, x \cdot b)
$$

holds for all $x \in \mathfrak{Q}$ and $a, b \in B$.

Finally, we require a trilinear inner composition $(a, b, c) \mapsto\langle a b c\rangle=$ $L(a, b) c$ to be given on $B$ making $B$ a triple system denoted by $B$ satisfying the following two axioms for all $a, b, c, d \in \mathbb{B}$ :

$$
\begin{gathered}
{[L(a, b), L(c, d)]=L(L(a, b) c, d)+L(c, L(b, a) d),} \\
\langle a b c\rangle-\langle c b a\rangle=\langle c a b\rangle-\langle a c b\rangle .
\end{gathered}
$$

A situation $\mathscr{R}, \sigma, f, \mathscr{B}$ which has the above properties is called admissible if the following relations hold for all $x \in \mathcal{Q}$ and $a, b, c, d \in \mathfrak{B}$ :

$$
\begin{gathered}
{[\sigma(x), L(a, b)]=L(x \cdot a, b)-L(a, x, b),} \\
L(a, b)-L(b, a)=\sigma(f(a, b)), \\
f(\langle a b c\rangle, d)+f(c,\langle a b d\rangle)=f(a, f(c, d) \cdot b) .
\end{gathered}
$$

2.2. REMARK. We consider the case where $\mathscr{U}=\mathrm{Fe}$ is the one-dimensional Jordan algebra over $F$. If $\sigma$ is any special unital representation of $\mathscr{Q}$ on the vector space $B$, we have $\sigma(\xi e) a=\xi a$ for all $\xi \in F$ and $a \in B$. The skew symmetric bilinear mappings $f: B \times B \mapsto \mathfrak{U}$ with (F) are exactly the skew symmetric bilinear forms on $B$.

Now, let $((B, D), \beta)$ be a Freudenthal triple system in the sense of $[9$, $\S 1,(1)-(4)]$ and let $((B, R), \beta)$ be a Faulkner triple system (cf. [3] for definition). Put

$$
f(a, b):=\beta(a, b) e \text { and }\langle a b c\rangle:=-2 D(a, b) c+1 / 4 \beta(a, b) c
$$


for $a, b, c \in B$. Then with $\mathcal{Q}$ and $\sigma$ as above we get an admissible situation थl, $\sigma, f,(B,\langle\rangle)$. Moreover, if $a, b, c \in B$ then

$$
\langle a b c\rangle=R(a, b) c-\beta(a, c) b+\beta(c, b) a .
$$

2.3. Example 1. Let $\mathscr{U}, \sigma, B$ be a special unital representation of the Jordan algebra $\mathscr{U}$ on the vector space $B$ which satisfies $\sigma(x) \sigma(y)=\sigma(y) \sigma(x)$ for all $x, y \in \mathfrak{U}$. Let $f: B \times B \mapsto \mathfrak{U}$ be a skew symmetric bilinear mapping such that (F) and $f(x, a, b)=f(a, x, b)$ hold for all $x \in \mathcal{Q}$ and $a, b \in B$. Then with the triple system defined by

$$
\langle a b c\rangle:=1 / 2(f(a, b) \cdot c-f(b, c) \cdot a+f(c, a) \cdot b),
$$

we get an admissible situation $\mathscr{\ell}, \sigma, f, \Re$. If $\mathscr{U}$ is one dimensional, this is exactly Example 1 in [3].

EXAMPLE 2. Let $\beta$ be a skew symmetric bilinear form on the vector space $B$ and $\mathscr{U}$ the Jordan algebra of symplectic symmetric endomorphisms of $B$, i.e.

$$
\mathfrak{U}=\left\{X \in \operatorname{End}_{F}(B) ; \beta(X a, b)=\beta(a, X b) \text { for } a, b \in B\right\}
$$

with multiplication $X \cdot Y=1 / 2(X Y+Y X)$. Define a special unital representation $\sigma$ of $\mathscr{U}$ by $\sigma(X) a:=X a$ for $X \in \mathfrak{U}$ and $a \in B$, and define for each pair $(a, b)$ of elements of $B$ an endomorphism of $B$ by $f(a, b) c:=\beta(a, c) b-$ $\beta(b, c) a$. It is immediate that $f(a, b)$ is in 2 for all $a, b \in B$, and an easy computation shows that the triple system $B$, defined by $\langle a b c\rangle:=\beta(c, a) b$ for $a, b, c \in B$ makes $\mathscr{Q}, \sigma, f, \Re$ an admissible situation. We remark that this example is a special case of the example considered in \$4.

2.4. Given an admissible situation $\mathscr{U}, \sigma, f, \mathbb{B}$, we make the vector space direct sum of $\mathscr{Q}$ and $B$ a triple system, denoted by $\mathfrak{X}$, which satisfies (1) and (2). For $x, y, z \in \mathfrak{Q}$ and $a, b, c \in \mathbb{B}$ we put $\langle x y z\rangle_{\mathfrak{A}}:=(x y) z+x(y z)-$ $y(x z)$, denote by \langle\rangle$_{B}$ the composition on $B$ and define

$$
\begin{aligned}
& \langle x y(z+c)\rangle:=\langle x y z\rangle_{y}-1 / 2 y \cdot(x \cdot a), \\
& \langle a b(z+c)\rangle:=-f(z \cdot a, b)+\langle a b c\rangle_{\mathscr{B}}, \\
& \langle x b(z+c)\rangle:=\langle a y(z+c)\rangle:=0
\end{aligned}
$$

for $x, y, z \in \mathfrak{Q}$ and $a, b, c \in \Re$. Clearly (1) and (2) are satisfied and with $L(x):=L(x, e), x \in \Re$, where $L(u, v) w:=\langle u v w\rangle$ for $u, v, w \in \mathfrak{T}$, we obtain $L(x) y=x y$ and $L(x) a=-1 / 2 x, a$ for $x, y \in \mathscr{U}$ and $a \in \mathbb{B}$. Moreover,

$$
\begin{gathered}
L(x, y)=L(x y)+[L(x), L(y)], \\
L(a, b)-L(b, a)=-2 L(f(a, b))
\end{gathered}
$$

for $x, y \in \mathbb{Q}$ and $a, b \in \mathscr{B}$. 
Next we define a bilinear mapping $D: \mathfrak{I} \times \mathfrak{T} \mapsto \operatorname{End}_{F}(\mathfrak{T})$ by

$$
\begin{aligned}
& D(x, y):=1 / 2(L(x, y)-L(y, x)), \\
& D(a, b):=-1 / 2(L(a, b)+L(b, a)), \\
& D(x, a):=D(a, x):=0
\end{aligned}
$$

for $x, y \in \mathscr{Q}$ and $a, b \in \Re$. Using (6) and (7) we obtain

$$
\begin{aligned}
& L(x, y)=L(x y)+D(x, y), \\
& L(a, b)=-L(f(a, b))-D(a, b)
\end{aligned}
$$

for $x, y \in \mathbb{Q}$ and $a, b \in \mathbb{B}$. Hence $L(\mathfrak{T}, \mathfrak{T})$ is the vector space sum of $L(\mathfrak{Z})$ and $D(\mathfrak{T} \mathfrak{T})$. This sum is direct since $D(x+a, y+b) e=0$ for all $x, y \in \mathfrak{Q}$ and $a, b \in \mathscr{D}$. Therefore we may define for $x \in \mathfrak{U}$ and $D \in D(\mathfrak{T}, \mathfrak{T})$

$$
(L(x)+D)^{*}:=L(x)-D
$$

to get an involutorial vector space automorphism ${ }^{*}$ of $L(\mathfrak{T}, \mathfrak{I})$. Note that

$$
\begin{aligned}
& L(x, y)^{*}=L(y, x), \\
& L(a, b)^{*}=-L(b, a)
\end{aligned}
$$

for all $x, y \in \mathscr{Q}$ and $a, b \in \Re$. Hence 1.3, a-c apply. In order to show that ${ }^{*}$ is an antiautomorphism of the Lie algebra $\Re(\mathfrak{I})$, we verify

$$
[N, L(u, v)]=L(N u, v)-L\left(u, N^{*} v\right)
$$

for $N \in \mathfrak{N}(\mathfrak{I})$ and $u, v \in \mathfrak{T}$. From this identity it is clear that the vector space $L(\mathfrak{T}, \mathfrak{T})$ is closed under the bracket multiplication, hence $\mathfrak{R}(\mathfrak{I})=L(\mathfrak{T}, \mathfrak{T})$, and an easy computation shows that (14) implies $\left[N_{1}, N_{2}\right]^{*}=\left[N_{2}^{*}, N_{1}^{*}\right]$ for $N_{i} \in L(\mathfrak{T}, \mathfrak{T})$. Now, (14) is an immediate consequence of the following three identities which we shall now prove (for the cases $u, v \in A$ applied to $A$ and $u, v \in B$ applied to $B$, first look at $N=L(a, b)$ and then at $N=L(x)$ ):

$$
\begin{gathered}
N^{*} f(a, b)+f(N a, b)+f(a, N b)=0, \\
N^{*}(x \cdot a)+(N x) \cdot a+x \cdot(N a)=0, \\
-2 L(x, f(a, b))=L(a, x \cdot b)-L(b, x \cdot a)
\end{gathered}
$$

for $N \in L(\mathfrak{T}, \mathfrak{T})$ and $a, b \in \mathfrak{B}$.

To verify (15) it is enough to consider the cases where $N=L(x, y)$ and $N=L(a, b)$ for $x, y \in \ell$ and $a, b \in \Re$. The latter case follows from $L(b, a) f(c, d)=f(a, f(c, d) . b), a, b, c, d \in \mathbb{B}$, which is immediate from (5) and (V3). It is clear from (6) that (15) holds for $N=L(x, y)$ if it holds for $N=$ $L(x)$, but this is what (F) says. 
Since $\sigma$ is s special representation of $\mathscr{U},(16)$ is satisfied for $N=L(x)$, $x \in \mathfrak{U}$. It remains to show (16) for $N \in D(\mathfrak{T}, \mathfrak{I})$. With $2 \bar{\sigma}(x):=-\sigma(x)$ we have

$$
[[\bar{\sigma}(x), \bar{\sigma}(y)], \bar{\sigma}(z)]=\bar{\sigma}(x(y z)-y(x z)) \text { for } x, y, z \in \vartheta
$$

which implies (16) for $N=D(x, y)$. Axiom (V1) yields $x .\langle a b c\rangle-\langle a b(x \cdot c)\rangle$ $=\langle(x, a) b c\rangle-\langle a(x . b) c\rangle$ for $x \in \mathscr{Q}$ and $a, b, c \in B$. Interchanging $a$ and $b$ implies

$$
x \cdot\langle b a c\rangle-\langle b a(x \cdot c)\rangle=\langle(x \cdot b) a c\rangle-\langle b(x \cdot a) c\rangle .
$$

Adding the last two identities and using (7) and (8), we get the desired result.

Since $\langle a b x\rangle=-f(x, a, b)$ for $x \in \mathcal{Q}$ and $a, b \in \mathbb{B}$ (see (5)), it follows from (F), (6) and (8) that (17) holds when applied to $x \in \mathcal{Q}$. Combining (7), (V1), (V2) and (16) we get

$$
\begin{aligned}
\langle a(x \cdot b) c\rangle- & \langle b(x \cdot a) c\rangle=\langle a(x \cdot b) c\rangle-\langle(x \cdot a) b c\rangle+f(x \cdot a, b) \cdot c \\
& =-x \cdot\langle a b c\rangle+\langle a b(x \cdot c)\rangle+f(x \cdot a, b) \cdot c \\
& =-x \cdot\langle a b c\rangle+\langle b a(x \cdot c)\rangle-f(b, a) \cdot(x \cdot c)+f(x \cdot a, b) \cdot c \\
& =\langle a b x\rangle \cdot c+f(a, b) \cdot(x \cdot c)+f(x \cdot a, b): c \\
& =-2\langle x f(a, b) c\rangle
\end{aligned}
$$

for $x \in \mathscr{Q}$ and $a, b, c \in B$. This completes the proof of (15)-(17).

We note that $(14)$ implies $D(\mathfrak{T}, \mathfrak{T})=\operatorname{Inder}(\mathfrak{I})$, where $\operatorname{Inder}(\mathfrak{T})$ is the Lie algebra of those endomorphisms $D \in L(\mathfrak{T}, \mathfrak{I})$, for which $D\langle u v w\rangle=\langle(D u) v w\rangle+$ $\langle u(D v) w\rangle+\langle u v(D w)\rangle$ holds for all $u, v, w \in \mathfrak{T}$, the so-called inner derivations of the triple system $\mathfrak{T}$.

The triple system $\mathfrak{I}$ and the involution * of the Lie algebra $L(\mathfrak{T}, \mathfrak{T})$ defined in the present section lead to the anticommutative algebra $\mathfrak{R}\left(\mathfrak{F}^{*}{ }^{*}\right)$ constructed in $\S 1.1$. We observe

THEOREM 1. $\mathcal{L}\left(\mathfrak{T}^{*}{ }^{*}\right)$ is a Lie algebra.

Proof. Following remark 1.3a, we must verify the identities (a)-(h) of $\S 1.1$. Now, (b) is immediate from the definition of \langle\rangle$_{\mathfrak{a}}$. Identity (a) is equivalent to (14), and (c) follows from (7), whereas (d) and (e) are special cases of definition (5). Finally, (f), (g) and (h) are equivalent to (16), (15) and (17) resp.

If $\mathfrak{T}$ is the triple system constructed as above from an admissible situation and ${ }^{*}$ is the involution defined by (11), we write $\mathfrak{L}(\mathfrak{I})$ for the Lie algebra $\mathfrak{R}(\mathfrak{S}, *)$.

3. Ideals in $\mathfrak{T}$ and $\mathfrak{R}(\mathfrak{T})$. Let $\mathfrak{Z}$ be a triple system over a field of char $\neq 3($ and $\neq 2)$ and $a$ a subspace of $B$. We call $a$ an ideal of $B$ if 
$\langle B \cap a\rangle \subset a,\langle B a B\rangle \subset a$ and $\langle a B B\rangle \subset a$. We call the triple, system $B$ simple if $\langle B \cap B\rangle \neq\{0\}$ and $B$ has no ideals different from $\{0\}$ and $B$.

For the present section we fix an admissible situation $\mathscr{U}, \sigma, f, B$ and denote the corresponding triple system constructed as in $\S 2.4$ by $\mathfrak{T}$.

3.1. With each ideal $t$ of $\mathfrak{T}$ there are associated two ideals of the Lie algebra $L(\mathfrak{I}, \mathfrak{T})$, namely

$$
\mathrm{n}_{\mathrm{t}}:=L(\mathfrak{T}, \mathrm{t})+L(\mathrm{t}, \mathfrak{T})
$$

and

$$
\mathfrak{m}_{\mathfrak{t}}:=\left\{N \in L(\mathfrak{T}, \mathfrak{I}) ; N(\mathfrak{T}) \subset \dot{\mathrm{t}} \text { and } N^{*}(\mathfrak{T}) \subset \mathrm{t}\right\} .
$$

Obviously the inclusion $\mathfrak{n}_{t} \subset \mathfrak{m}_{t}$ holds and a simple computation shows that, if $\mathfrak{h}$ is any ideal of $L(\mathfrak{Y}, \mathfrak{T})$, the vector space direct sum $\mathfrak{l}=\mathfrak{G} \oplus t \oplus \mathfrak{t}$ is an ideal of $\mathfrak{\Omega}(\mathfrak{T})$ if and only if

$$
n_{t} \subset \mathfrak{G} \subset m_{t} \cdot
$$

We define the projections $R, S$ and $\bar{S}$ of $\mathfrak{R}(\mathfrak{T})$ onto the subspaces $L(\mathfrak{T}, \mathfrak{T})$, $\mathfrak{T}$ and $\overline{\mathfrak{T}}$ resp., and the projections $P$ and $Q$ of $\mathfrak{T}$ onto the subspaces $\mathcal{U}$ and $\mathfrak{B}$ of $\mathfrak{T}$ resp. by

$$
\begin{gathered}
R(X):=N, \quad S(X):=u, \quad \bar{S}(X):=\bar{v} ; \\
P(u):=x, \quad Q(u):=a
\end{gathered}
$$

whenever $X=N+u+\bar{v}$ and $u=x+a, N \in L(\mathfrak{T}, \mathfrak{T}), x \in \mathscr{U}$ and $a \in \mathfrak{H}$.

If $a$ is any ideal of $\mathfrak{R}(\mathfrak{T})$ and $t$ is any ideal of $\mathfrak{T}$, then $R(a), S(a), \bar{S}(\dot{a})$, $P(t)$ and $Q(t)$ are ideals of $L(\mathfrak{T}, \mathfrak{T}), \mathfrak{T}, \overline{\mathfrak{T}}, \mathfrak{Q}, \mathfrak{B}$ resp. For each $u=x+a \in$ $\mathrm{t}$ we have $L(e) u-u=-(3 / 2) a$, which implies $a \in t$, hence $u-a=x \in t$. It follows that

$$
t=P(t) \oplus Q(t)
$$

for any ideal $t$ of $\mathfrak{T}$.

If $a$ is any ideal in $\mathfrak{R}(\mathfrak{S})$ and $X=N+u+\dot{v} \in a, u=x+a, v=y+b$, then $[e,[e, X]]=-2 y$ and $[\bar{e},[\bar{e}, X]]=-2 \bar{x}$ imply $x, y \in a$. Moreover, $[L(e), X]=x-1 / 2 a-\bar{y}+1 / 2 \bar{b} \in a$ yields $w:=a-\bar{b} \in a$. Since $[e, w]=$ $-2 \bar{a}$ and $[\bar{e}, w]=2 b$, we have $a, b \in a$. Finally we have $N \in a$ since $N=$ $X-u-\bar{v}$ and $u, \bar{v} \in a$. This and $\bar{S}(a)=\overline{S(a)}$ imply

$$
a=R(a) \oplus S(a) \oplus \overline{S(a)} \text {. }
$$

Using (18) we conclude that $a=\{0\}$ if and only if $S(a)=\{0\}$, and that $a=$ $\mathfrak{L}(\mathfrak{T})$ if and only if $S(a)=\mathfrak{T}$. Since $[L(e), x+a]=L(e)(x+a)=x-1 / 2 a$ for $x \in \mathscr{Q}$ and $a \in \mathfrak{B}$ we see that $L(\mathfrak{T}, \mathfrak{T})=\{0\} \quad$ if and only if 
$[R(\mathfrak{T}), \mathfrak{R}(\mathfrak{T})]=\{0\}$. Hence we observe

THEOREM 2. $\{(\mathfrak{I})$ is simple if and only if $\mathfrak{T}$ is simple.

3.2. For the rest of this section we assume that $f$ is not the zero mapping. We saw that each ideal of $\mathfrak{F}$ can be written as $a \oplus \mathfrak{b}$, where $a$ is an ideal of $\mathfrak{A}$ and $\mathfrak{b}$ is an ideal of $\mathscr{B}$. Conversely given ideals $a, \mathfrak{b}$ of $\mathscr{U}$ and $\mathscr{B}$ resp., the sum $a \oplus \mathfrak{b}$ is an ideal of $\mathfrak{T}$ if and only if

$$
\sigma(\mathfrak{Q}) \mathfrak{b} \subset \mathfrak{b}, \quad \sigma(a) B \subset b, \quad f(b, B) \subset a .
$$

It follows immediatly that $\operatorname{ker} \sigma \oplus\{0\},\langle\operatorname{im} f\rangle \oplus B$ and $\{0\} \oplus\{a \in B ; f(a, B)$ $=\{0\}\}$ are ideals of $' \mathfrak{T}$, where $\langle\mathrm{im} f\rangle$ denotes the subspace of $\mathscr{U}$ generated by $f(a, b), a, b \in \mathbb{B}$. If $\mathfrak{T}$ is simple we conclude that $\sigma$ is faithful, $f$ is nondegenerate and $\langle\operatorname{im} f\rangle=\imath$. Moreover, we prove

THEOREM 3. $\mathfrak{T}$ is simple if and only if $\mathscr{B}$ has no $\sigma(\mathfrak{U})$-invariant ideals different from $\{0\}$ and $\mathfrak{B}, \sigma$ is faithful and $\langle\operatorname{im} f\rangle=\mathfrak{Q}$.

Proof. Suppose $\mathfrak{T}$ is not simple and $t$ is an ideal of $\mathfrak{T}$ such that $\{0\}$ $\neq t \neq \mathfrak{T}$. Write $t=a \oplus b$ where $a$ and $b$ are suitable ideals of $\mathscr{V}$ and $B$ resp. Then (21) yields $\sigma(\mathfrak{U}) \mathfrak{b} \subset \mathfrak{b}$. If $\{0\}$ and $B$ are the only $\sigma(\mathscr{V})$-invariant ideals of $B$ we obtain that either $\mathfrak{b}=\{0\}$ or $b=B$. If $\sigma$ is faithful (21) shows that $b=\{0\}$ implies $a=\{0\}$, hence $t=\{0\}$. Using (21) again we see that $\langle\operatorname{im} f\rangle=\mathfrak{Z}$, and $\mathfrak{b}=\mathfrak{B}$ implies $a=\mathfrak{Z}$, hence $t=\mathfrak{T}$. Thus in any case we get a contradiction to our assumption on $t$.

3.3. We consider the important case, where the Jordan algebra $\mathfrak{U}$ is simple. Recall that $\{0\} \oplus\{a \in \mathfrak{B} ; f(a, \mathbb{B})=\{0\}\}$ is an ideal of $\mathfrak{T}$ and that $\{0\} \oplus \mathbb{B}$ is not an ideal of $\mathfrak{T}$ since $f \neq 0$. Hence $\mathfrak{T}$ simple implies $f$ nondegenerate. It is an immediate consequence of (21) that any ideal $\neq \mathfrak{T}$ of $\mathfrak{T}$ can be written as $\{0\} \oplus \mathscr{b}$ where $\mathfrak{b}$ is an ideal of $B$ different from $B$, such that $f(\mathfrak{b}, B)=\{0\}$. Hence $f$ nondegenerate implies $b=\{0\}$. Thus we observe

Proposition. If the Jordan algebra 2 it is simple, then $\mathfrak{T}$ is simple if and only if $f$ is nondegenerate.

4. Lie algebras of type $B, C, D$. Let $U$ be a vector space of finite dimension over a field $F$ of characteristic not two and $\lambda$ a bilinear form on $U$. We denote by 2 the Jordan algebra of those endomorphisms $X$ of $U$, for which $\lambda(X a, b)=\lambda(a, X b)$ for $a, b \in U$. The product of two such endomorphisms is defined as usual by $X \cdot Y=1 / 2(X Y+Y X)$. Let $W$ be another vector space over $F$ of finite dimension and $\mu$ a bilinear form on $W$. We choose a basis $a_{1}, \cdots, a_{r}$ of $U$ and a basis $b_{1}, \cdots, b_{s}$ of $W$ and define a representation 
$\sigma$ of $थ$ on the vector space $V:=U \otimes_{F} W$ by

$$
\sigma(X)\left(a_{i} \otimes b_{j}\right):=X a_{i} \otimes b_{j}
$$

for $X \in \mathscr{Q}$ and $1 \leqslant i \leqslant r, 1 \leqslant j \leqslant s$. This representation is clearly faithful and unital special. Now, we define a trilinear inner composition on $V$ by

$$
\begin{aligned}
\left\langle\left(a_{i} \otimes b_{j}\right)\left(a_{k} \otimes b_{l}\right)\left(a_{m} \otimes b_{n}\right)\right\rangle:= & \lambda\left(a_{i}, a_{k}\right) \mu\left(b_{n}, b_{l}\right) a_{m} \otimes b_{j} \\
& -\lambda\left(a_{k}, a_{m}\right) \mu\left(b_{l}, b_{j}\right) a_{i} \otimes b_{n} \\
& +\lambda\left(a_{k}, a_{i}\right) \mu\left(b_{n}, b_{j}\right) a_{m} \otimes b_{l}
\end{aligned}
$$

for $1 \leqslant i, k, m \leqslant r$ and $1 \leqslant j, l, n \leqslant s$. It is immediate that for $a, b \in V$ the endomorphism $f(a, b):=L(a, b)-L(b, a)$ is in $\mathscr{U}$ if (a) $\lambda$ is skew symmetric and $\mu$ is symmetric, or (b) $\lambda$ is symmetric and $\mu$ is skew symmetric. An elementary proof shows that in both cases the situation $थ, \sigma, f,(V,\langle\rangle)$ is admissible. If $\lambda$ and $\mu$ are not the zero forms and are nondegenerate, then $f$ is nondegenerate. Using the proposition of $\S 3$, we observe that the Lie algebra $\mathfrak{Z ( \mathfrak { I } )}$ is simple since $\mathfrak{Q}$ is simple (cf. [1, Satz 3.2, p. 189]). In the case (a) $\mathfrak{L}(\mathfrak{I})$ is of type $B_{r+t}$ if $s=2 t+1$ and of type $D_{r+t}$ if $s=2 t$. If (b) holds we have $s=2 t$ and $\&(\mathfrak{I})$ is of type $C_{r+t}$.

REMARK. There is another way to describe the above situation $\mathcal{Q}, \sigma, f$, $(V,\langle\rangle)$, which is due to U. Hirzebruch: Let $V$ be the vector space of all $r$ by $s$ matrices over $F$ and $A \mapsto \bar{A}$ a $F$-linear mapping from $V$ to the vector space of all $s$ by $r$ matrices over $F$, such that

$$
\overline{A \bar{B} C}=-\bar{C} B \bar{A}
$$

for $A, B, C \in V$. Denote by $\overline{\mathscr{Q}}$ the set of those $r$ by $r$ matrices $X$ over $F$, for which $\overline{X A}=\bar{A} X$ is satisfied for all $A \in V$. It follows immediately that $\overline{\mathfrak{U}}$ is a Jordan algebra under the multiplication $X \cdot Y=1 / 2(X Y+Y X)$, where $X Y$ is the ordinary matrix product. $\overline{\mathscr{U}}$ acts on $V$ by matrix multiplication. Denote this action by $\bar{\sigma}$. Put

$$
f(A, B):=A \bar{B}-B \bar{A} \text { and }\langle A B C\rangle:=A \bar{B} C+C \bar{B} A+C \bar{A} B
$$

for $A, B, C \in V$. The situation $\overline{\mathscr{U}}, \bar{\sigma}, f,(V,\langle>)$ is then admissible.

We specialise this situation by letting $\bar{A}:=M A^{t} N, A \in V$ where $A^{t}$ is the transpose of $A, M$ is an $r$ by $r$ matrix over $F$ and $N$ is an $s$ by $s$ matrix over $F$, such that either $M$ is skew symmetric and $N$ is symmetric or $M$ is symmetric and $N$ is skew symmetric. Further we let $\mathfrak{U}$ be the subalgebra of $\overline{\mathfrak{U}}$ formed by those elements of $\overline{\mathscr{U}}$ which commute with $N^{t}$. We denote the restriction of $\bar{\sigma}$ to $\mathscr{U}$ by $\sigma$. It is easily seen that $f(A, B) \in \mathscr{U}$ for all $A, B \in V$, 
and a simple computation shows that this specialisation of the above situation exactly yields those triple systems which led us to the Lie algebras of type $B, C$ and $D$.

5. Triple systems defined by symplectic representations of Jordan algebras.

5.1.1. Let $थ$ be a finite-dimensional Jordan algebra over a field $F$ of characteristic not two with unit element $e \neq 0$ and $\alpha$ a nondegenerate symmetric bilinear form on $थ, \alpha \neq 0$ such that

$$
\alpha(x y, z)-\alpha(x, y z)=0
$$

for $x, y, z \in \mathscr{U}$. Let $\sigma$ be a faithful special unital representation of 2 on the vector space $B$ over $F$ which is symplectic with respect to the nondegenerate skew symmetric bilinear form $\beta \neq 0$ on $B$, that means

$$
\beta(x, a, b)=\beta(a, x, b)
$$

for $x \in \mathcal{Q}$ and $a, b \in B$, where $x, a$ stands for $\sigma(x) a$.

5.1.2. Let $D$ be a finite-dimensional Lie algebra over $F$ and $\mu \neq 0$ a nondegenerate symmetric associative bilinear form on (associative means $\left.\mu\left(D_{1},\left[D_{2}, D_{3}\right]\right)=\mu\left(\left[D_{1}, D_{2}\right], D_{3}\right)\right)$. Assume that a faithful symplectic representation $\rho$ of $D$ on the symplectic space $(B, \beta)$ is given, such that

$$
\begin{gathered}
{[\sigma(\mathfrak{Z}), \sigma(\mathfrak{U})] \subset \rho(\mathfrak{P}),} \\
{[\sigma(\mathfrak{Y}), \rho(\mathfrak{J})] \subset \sigma(\mathfrak{Y}),} \\
\alpha^{\prime}(\sigma(x),[\rho(D), \sigma(y)])=\mu^{\prime}(\rho(D),[\sigma(y), \sigma(x)]) \\
=\alpha^{\prime}(\sigma(y),[\sigma(x), \rho(D)])
\end{gathered}
$$

for $x, y \in \mathcal{U}$ and $D \in \mathcal{\vartheta}$, where $\alpha^{\prime}$ and $\mu^{\prime}$ are defined by

$$
\alpha^{\prime}(\sigma(x), \sigma(y)):=\alpha(x, y), \quad \mu^{\prime}\left(\rho\left(D_{1}\right), \rho\left(D_{2}\right)\right):=\mu\left(D_{1}, D_{2}\right) .
$$

5.2.1. For a first set of examples, which plays a role later on choose a Lie algebra $\supseteqq$ with nondegenerate Killing form $\mu$ and symplectic representation $\rho$ on the nonsingular symplectic space $(B, \beta)$. Let $\mathfrak{U}=F e$ be the one-dimensional Jordan algebra over $F$. Then there is exactly one special unital representation $\sigma$ of $थ$ on $B$ given by $\sigma(\xi e) a=\xi a$ for $\xi \in F$ and $a \in B$. Now, (23)-(26) are satisfied. Since every bilinear form $\alpha$ on $\mathscr{U}$ satisfies $\alpha(\xi e, \eta e)=\alpha(e, e) \xi \eta$ for $\xi, \eta \in F$, the identity (22) is clearly satisfied.

5.2.2. A more important set of examples which leads to the Lie algebras of type $F_{4}$ and $E_{8}$ is the following: Let $\mathscr{U}, \alpha, \sigma, B, \beta$ be given as in 5.1.1. Put $\vartheta:=[\sigma(\mathfrak{U}), \sigma(\mathfrak{Q})]$ and $\rho:=\mathrm{id}_{B}$. Then $\rho$ is a faithful symplectic representation of the Lie algebra $D$ on the symplectic space $(B, \beta)$ and the outer terms of identity (26) obviously agree. Now, $\alpha$ induces a nondegenerate symmetric asso- 
ciative bilinear form $\mu$ on $\vartheta$, which is uniquely determined by

$$
\mu\left([\sigma(x), \sigma(y)],\left[\sigma\left(x^{\prime}\right), \sigma\left(y^{\prime}\right)\right]\right)=-4\left(\alpha\left(x x^{\prime}, y y^{\prime}\right)-\alpha\left(x y^{\prime}, x^{\prime} y\right)\right)
$$

for $x, x^{\prime}, y, y^{\prime} \in$ थ. Clearly 5.1 .2 is also satisfied.

5.3. From now on let the characteristic of $F$ be different from two and three. In order to construct an admissible situation (see 2.1) from the data of 5.1.1 and 5.1.2, we define a bilinear mapping $f: B \times B \mapsto \mathcal{Q}$ by

$$
\alpha(x, f(a, b))=2 \beta(x \cdot a, b)
$$

for $x \in \mathscr{Q}$ and $a, b \in B$, and a bilinear mapping $D: B \times B \mapsto \operatorname{End}_{F}(B)$ by

$$
\mu^{\prime}(\rho(D), D(a, b))=-\beta(\rho(D) a, b)
$$

for $D \in \supseteqq$ and $a, b \in B$. Next we put

$$
\langle a b c\rangle:=1 / 2 f(a, b) \cdot c-D(a, b) c
$$

for $a, b, c \in B$, and this makes $B$ a triple system which we denote by $B$.

THEOREM 4. $\mathscr{R}, \sigma, f, B$ is an admissible situation if and only if

$$
D(a, b) c-D(c, b) a=1 / 2 f(a, b) \cdot c+1 / 2 f(b, c) \cdot a-f(c, a) \cdot b
$$

or equivalently $\langle a b c\rangle-\langle c b a\rangle=f(c, a) \cdot b$ for $a, b, c \in \mathbb{B}$.

If $(31)$ is satisfied the corresponding Lie algebra $\mathfrak{L}(\mathfrak{I})$ is simple if the Jordan algebra $\mathfrak{U}$ is simple.

Proof. It follows immediately from (24) and (25) that $\Re:=\sigma(\Re) \oplus$ $\rho(\supseteqq)$ is a subalgebra of the Lie algebra of all endomorphisms of $B$. Using (26) we obtain a nondegenerate symmetric associative bilinear form $\lambda$ on $\Re$ by

$$
\lambda\left(\sigma(x)+\rho(D), \sigma\left(x^{\prime}\right)+\rho\left(D^{\prime}\right)\right):=\alpha\left(x, x^{\prime}\right)+\mu\left(D, D^{\prime}\right)
$$

for $x, x^{\prime} \in \mathfrak{Q}$ and $D, D^{\prime} \in \mathfrak{\vartheta}$. Obviously we have

$$
\lambda(N, L(a, b))=\beta(N a, b)
$$

for all $N \in \mathfrak{N}$ and $a, b \in \Re$. Now we are ready to establish our axioms. First, we get $(F)$ as a simple consequence of (22) and (28). Since $\sigma$ and $\rho$ are symplectic with respect to $\beta,(30)$ yields $\beta(L(a, b) c, d)=-\beta(c, L(b, a) d)$. The associativity of $\lambda$ and (32) imply

$$
\lambda(N,[L(a, b), L(c, d)])=\beta(N L(a, b) c, d)-\beta(L(a, b) N c, d)
$$

for $N \in \Re$ and $a, b, c, d \in \Re$. These two identities imply (L1). Obviously (L2) and (V2) follow from (31) and (30) resp. Using again the associativity of $\lambda$ we obtain (V1). In order to check (V3) we first prove

$$
2[\sigma(x), D(a, b)]=-\sigma(f(x . a, b)+f(a, x . b)) \text { for } x \in \mathfrak{Q} \text { and } a, b \in \Re \text {. }
$$


The associativity of $\lambda$ implies

$$
\begin{aligned}
2 \lambda(\sigma(y)+ & \rho(D),[\sigma(x), D(a, b)])=2 \lambda([\sigma(y), \sigma(x)], D(a, b)) \\
& =-2 \beta(y \cdot(x, a), b)+2 \beta(y, a, x, b) \\
& =-\alpha(y, f(x, a, b))+\alpha(y, f(a, x, b)) \\
& =\lambda(\sigma(y)+\rho(D),-\sigma(f(x, a, b))+\sigma(f(a, x, b)))
\end{aligned}
$$

for $x, y \in \mathfrak{U}, D \in \mathfrak{D}$.

Since $\lambda$ is nondegenerate, this implies the desired identity. We shall now show (V3). For any $x \in \mathfrak{U}$ we have

$$
\begin{aligned}
& \alpha(x, f(\langle a b c\rangle, d))+\alpha(x, f(c,\langle a b d\rangle)) \\
&= 2 \beta(x \cdot\langle a b c\rangle, d)+2 \beta(x \cdot c,\langle a b d\rangle) \\
&= \beta(x \cdot(f(a, b) \cdot c), d)-2 \beta(x \cdot(D(a, b) c), d) \\
&+\beta(f(a, b) \cdot(x \cdot c), d)+2 \beta(D(a, b)(x, c), d) \\
&= 2 \beta((x f(a, b)) \cdot c, d)+\beta(f(x, a, b), c, d)-\beta(f(a, x, b) \cdot c, d) \\
&= 2 \beta(f(x, a, b) \cdot c, d)=\alpha(f(x \cdot a, b), f(c, d)) \\
&=-2 \beta(f(c, d) \cdot b, x \cdot a)=\alpha(x, f(a, f(c, d), b)), \quad a, b, c, d \in \Re .
\end{aligned}
$$

Since $\alpha$ is nondegenerate this yields (V3).

Suppose (31) is satisfied and 2 is simple. Since $\beta$ is nondegenerate, $f$ is also. Using the proposition of $\S 3$, we conclude that $\mathfrak{R}(\mathfrak{Y})$ is simple.

5.4. Following 5.2.1 we give the data $\supseteq, \rho, B, \beta$ which lead to Lie algebras of type $G_{2}$ : Let $D$ be the Lie algebra of 2 by 2 matrices over an arbitrary field of characteristic not two and three with trace zero. Let $B$ be the four-dimensional vector space over the same field with basis $e_{0}, e_{1}, e_{2}, e_{3}$. We define the representation $\rho$ of $D$ on the basis

$$
H=\left(\begin{array}{rr}
1 & 0 \\
0 & -1
\end{array}\right), \quad E=\left(\begin{array}{ll}
0 & 1 \\
0 & 0
\end{array}\right), \quad F=\left(\begin{array}{ll}
0 & 0 \\
1 & 0
\end{array}\right)
$$

of 9 by

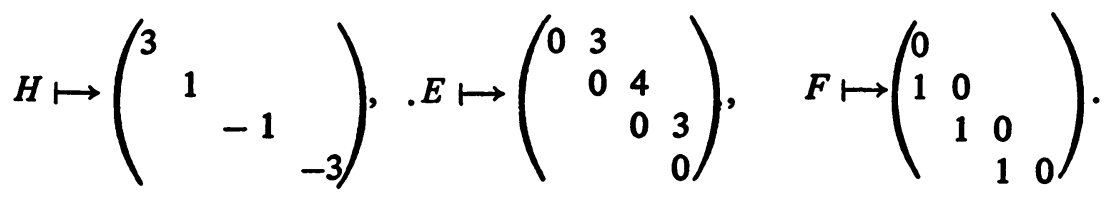

With the notation $v_{i}:=3-2 i \quad(0 \leqslant i \leqslant 3), \mu_{i}:=i(4-i) \quad(1 \leqslant i \leqslant 4)$ and $e_{-1}:=e_{4}:=0$, the representation $\rho$ can be written as

$$
\rho(H) e_{i}=v_{i} e_{i}, \quad \rho(E) e_{i}=\mu_{i} e_{i-1}, \quad \rho(F) e_{i}=e_{i+1}
$$


for $0 \leqslant i \leqslant 3$. This representation is clearly faithful. It is symplectic with respect to the nondegenerate skew symmetric bilinear form $\beta$, defined by the matrix

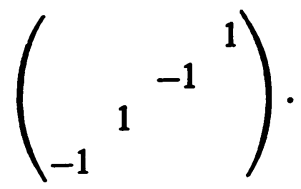

We define the bilinear form $\alpha$ by $\alpha(e, e)=-8 / 3$ which yields $f(a, b)=$ $-(3 / 4) \beta(a, b) e$ ( $e$ as in 5.2.1).

In order to check (31) we note that, if $\mu$ denotes the Killing form of $D$, the relations $\mu(H, H)=8, \mu(E, F)=4, \mu(H, E)=\mu(H, F)=\mu(E, E)=\mu(E, F)=0$ holds. Using (29) we obtain for $0 \leqslant i, j, k \leqslant 3$

$$
D\left(e_{i}, e_{j}\right) e_{k}=1 / 2 v_{i} v_{k} \beta\left(e_{i}, e_{j}\right) e_{k}+\mu_{k} \beta\left(e_{i+1}, e_{j}\right) e_{k-1}+\mu_{i} \beta\left(e_{i-1}, e_{j}\right) e_{k+1} .
$$

Now, an easy computation establishes (31).

5.5. We shall now give the construction of Lie algebras of type $F_{4}$ and $E_{8}$ according to 5.2.2. What we need is a faithful symplectic special unital representation of a finite-dimensional Jordan algebra $\mathcal{U}$ with unit element $e \neq 0$ on a finitedimensional nonsingular symplectic space, where $\mathfrak{Q}$ admits a nondegenerate symmetric associative bilinear form.

Let $V$ be a vector space of finite dimension $n$ over a field $F$ of characteristic not two or three. Let $q$ be a quadratic form on $V$ with associated symmetric bilinear form $\alpha$, defined by

$$
\alpha(x, y)=1 / 2(q(x+y)-q(x)-q(y)) \text { for } x, y \in V .
$$

We denote the Clifford algebra of $q$ by $\mathfrak{E}(V, q)$ or $\mathfrak{E}$. The subspace $\mathcal{Q}:=$ $\mathrm{Fe} \oplus V$, e the unit element of $\sqrt{ }$, becomes a Jordan algebra by

$$
(\xi e+x)(\eta e+y):=(\xi \eta+\alpha(x, y)) e+\eta x+\xi y
$$

for $\xi, \eta \in F$ and $x, y \in V$, of which $\mathcal{S}$ is the special unital universal envelope (cf. [5, p. 74, example (4)]). We extend $\alpha$ to a nondegenerate symmetric bilinear form $\alpha^{\prime}$ on $थ$ by

$$
\alpha^{\prime}(e, e):=1, \quad \alpha^{\prime}(e, x):=\alpha^{\prime}(x, e):=0, \quad \alpha^{\prime}(x, y):=\alpha(x, y)
$$

for $x, y \in V$. Immediately $\alpha^{\prime}$ satisfies (22). Since $\alpha$ is nondegenerate the Jordan algebra 2 is simple (cf. [1, Satz 5.7, p. 197]).

Suppose now that $q$ is of maximal Witt index $r>2$. Hence $n=2 r$ or $n=2 r+1$. We choose maximal totally isotropic subspaces $N$ and $P$ of $V$ satisfying $N \cap P=\{0\}$, a basis $x_{1}, \cdots, x_{r}$ of $N$ and a basis $x_{r+1}, \cdots$, 
$x_{2 r}$ of $P$ such that

$$
\alpha\left(x_{i}, x_{j}\right)=\delta_{i+r, j} \quad(1 \leqslant i, j \leqslant 2 r) .
$$

If $n=2 r$ clearly $V=N \oplus P$ holds. Let $n=2 r+1$. We assume that $2(-1)^{r} D$ is a square in $F, D$ being the discriminant of $q$. Then there exists a vector $x_{n}$ in $V$ such that

$$
\alpha\left(x_{i}, x_{n}\right)=\delta_{i, n} \quad(1 \leqslant i \leqslant n) .
$$

Note that $V=N \oplus P \oplus F x_{n}$.

Recall that the elements of the form $x_{i_{1}} \cdots x_{i_{m}}, 1 \leqslant i_{1}<\cdots<i_{m} \leqslant n$, form a basis of $\mathfrak{C}$. We denote such an element by $x_{M}$ if $M=\left\{i_{1}, \cdots, i_{m}\right\}$. Let $B$ be the subspace of $\subseteq$ spanned by the $x_{M}, M \subset R:=\{1, \cdots, r\}$. In order to define a representation $\tau$ of $\mathcal{E}$ on $B$ it is enough to define $\tau\left(x_{i}\right) x_{M}$ for $1 \leqslant i \leqslant n$ and all $M \subset R$. We put

$$
\tau\left(x_{i}\right) x_{M}:=\xi_{i} x_{i} x_{M} \quad(1 \leqslant i \leqslant n, M \subset R)
$$

where $\xi_{i}:=1$ if $i \in R$ or $i-r \in M ; \xi_{i}:=0$ if $i-r \in R \backslash M$; if $n=2 r+1$ the factor $\xi_{n}$ is defined by $(-1)^{m}, m$ being the number of elements of $M$.

We note that this representation is a spin representation of $\mathfrak{E}$. The description above can easily be deduced from the definitions of $[2, \mathrm{p} .70]$.

Since $\tau$ is a faithful representation of $\sqrt{ }$ and $\Subset$ is the special unital universal envelope of $\mathscr{U}$, the restriction of $\tau$ to $\mathcal{U}$ is a faithful special unital representation of $\mathscr{U}$ on the vector space $B$ which we denote by $\sigma$.

It is shown in $[2, \S 3.2]$ that there exists a nondegenerate bilinear form $\beta$ on $B$ satisfying $\beta(\tau(x) a, b)=\beta(a, \tau(x) b)$ for all $x \in V$ and $a, b \in B$. It is easily seen that this implies

$$
\beta\left(x_{A}, x_{B}\right) \neq 0 \text { if and only if } A \cup B=R \text { and } A \cap B=\varnothing
$$

for $A, B \subset R$. Moreover, $\beta$ is skew symmetric if $r \equiv 2$ or $3 \bmod 4$. Therefore we suppose that this condition on $r$ is always satisfied. Hence $\sigma$ is symplectic.

Now, let $\mathcal{Q}, \sigma, f, \mathfrak{B}$ be constructed as in 5.3 from the data $\mathcal{U}, \sigma, B, \beta$ defined above and those of 5.2.2.

TheOREM 5. The situation $थ, \sigma, f, B$ is admissible if and only if the dimension of $V$ is either 6 or 13. In the former case the corresponding Lie algebra $\mathfrak{l}(\mathfrak{I})$ is of type $F_{4}$ whereas $\mathfrak{I}(\mathfrak{I})$ is of type $E_{8}$ in the latter case.

Proof. According to Theorem 4 we must show that (31) holds if and only if $n=6$ or $n=13$. The rest of the theorem is then clear because $\mathfrak{R}(\mathfrak{T})$ is 
simple and has dimension 52 if $n=6$, it has dimension 248 if $n=13$. Now, using (33)-(36) both sides of (31) can be computed from the definitions (28) and (29). Note that if (31) holds for $b=e, e$ the unit element of $\sqrt{ }$, then (31) holds for all $b \in \mathbb{B}$. This can be proved by an easy induction on the number of elements of $M$ if $b=x_{M}$. The statement that $n=6$ or $n=13$ is necessary for (31) to be satisfied follows from the case $a=x_{R}, b=c=e$.

\section{REFERENCES}

1. H. Braun and M. Koecher, Jordan-Algebren, Die Grundlehren der math. Wissenschaften in Einzeldarstellungen mit besonderer Berücksichtigung der Anwendungsgebiete, Band 128, Springer-Verlag, Berlin and New York, 1966. MR 34 \#4310.

2. C. C. Chevalley, The algebraic theory of spinors, Columbia Univ. Press, New York, 1954. MR 15, 678.

3. J. R. Faulkner, A construction of Lie algebras from a class of ternary algebras, Trans. Amer. Math. Soc. 155 (1971), 397-408. MR 45 \#3494.

4. H. Freudenthal, Beziehungen der $E_{7}$ und $E_{8}$ zur Oktavenebene. II, Nederl. Akad. Wetensch. Proc. Ser. A 57 = Indag. Math. 16 (1954), 363-368.

5. U. Hirzebruch, Über eine Klasse von Lie-Algebren, J. Algebra 11 (1969), 461-467. MR 38 \#5869.

6. N. Jacobson, Structure and representations of Jordan algebras, Amer. Math. Soc. Colloq. Publ., vol. 39, Amer. Math. Soc., Providence, R. I., 1968. MR 40 \#4330.

7. M. Koecher, Imbedding of Jordan algebras into Lie algebras. I, Amer. J. Math. 89 (1967), 787-816. MR 35 \#5480.

8. K. Meyberg, Jordan-Tripelsysteme und die Koecher-Konstruktion von Lie-Algebren, Math. Z. 115 (1970), 58-78. MR 41 \#8482.

9. - Eine Theorie der Freudenthalschen Tripelsysteme. I, Nederl. Akad. Wetensch. Proc. Ser. A 71 = Indag. Math. 30 (1968), 162-174. MR 37 \#1429.

MATHEMATISCHES INSTITUT, UNIVERSITÄT MÜNSTER, MÜNSTER, FEDERAL REPUBLIC OF GERMANY

Current address: G. H. Siegen Lehrstuhl für Mathematik III, 993 Hüttental-Weidenan, Hölderstrasse 3, Federal Republic of Germany 